\title{
Observational Study of the Effect of Consumption of a Magnesium- and B Vitamin-Enriched Gingerbread on Cognitive Well-being (Mood, Stress, and Sleep)
}

\author{
Francois-André Allaert ${ }^{1^{*}}$, Romain Schueller ${ }^{2}$, Florent Herpin ${ }^{2}$ and Florian Rigaudier ${ }^{2}$ \\ ${ }^{1}$ ESC Chair of Medical Evaluation and Cen Nutriment Dijon, France \\ ${ }^{2}$ CEN Nutriment Dijon, France
}

*Corresponding author: Allaert FA, ESC Chair of Medical Evaluation and Cen Nutriment Dijon, France, Tel: 0380680511; E-mail: allaert@cenbiotech.com

Received date: 22 June, 2017; Accepted date: 03 July, 2017; Published date: 10 July, 2017

Copyright: ( 2017 Allaert FA, et al. This is an open-access article distributed under the terms of the Creative Commons Attribution License, which permits unrestricted use, distribution, and reproduction in any medium, provided the original author and source are credited.

\begin{abstract}
Objective: According to European Commission Regulation No 432/2012, the incorporation into food of minerals and oligo-elements which are officially linked to a health claim means the "health claim" can be made for the food too. Nevertheless, their introduction into various food matrices can potentially influence their effectiveness. The objective of this study was to confirm the effect on daily mood, stress and sleep of a daily intake of magnesium- and $B$ vitamin-enriched gingerbread for 3 weeks at the required quantities determined by EFSA ( $30 \%$ of RDAs).

Methods: Selection criteria: men or women, aged over 18 years and under 70 years of age, complaining of a mood or stress rated by an MSP-9 scale. Evaluation criteria: MSP-9, Brief Mood Introspection Scale (BMIS) and Spiegel scale for sleep. Daily data collection on a Smartphone CRF (NursTrial System) for 7 days before the consumption of gingerbread and during the last week of consumption.

Results: 185 persons aged $39 \pm 12$ years and mostly female were included in the study. The results show significant reductions in stress assessed by MSP-9, which significantly decreased from $42.6 \pm 8.5$ to $30.9 \pm 8.4$ $(p<0.0001)$, a $26 \%$ improvement in BMIS-assessed mood from $38.6 \pm 6.6$ to $47.2 \pm 7.3(p<0.0001)$, an improvement of $25 \%$, and quality of sleep evaluated by the Spiegel scale, which increased from $16.2 \pm 3.5$ to $19.3 \pm 3.6$ $(p<0.0001)$, corresponding to an improvement of $23 \%$. Some $35.1 \%$ of the subjects declared they were rather satisfied and $50.3 \%$ they were satisfied or very satisfied.
\end{abstract}

Keywords: Mood; Stress; Sleep; Magnesium; B vitamins

\section{Introduction}

Many studies have shown the adverse effects of stress on cognitive performance and quality of sleep [1-6]. Extended exposure to stress in particular can impair memory $[7,8]$ and people in stressful situations exhibit more memory and attention disorders [9-11].

Doctors and pharmacists used to suggest patients take medicinal products that are no longer reimbursed because ineffective. The lack of credibility of such products means that a 'cognitive' gingerbread enriched in ingredients for which an official EFSA health claim is made and whose properties have been confirmed by a real-life clinical observational study could be of interest to the many people looking for such products to cope with the cognitive disorder they experience daily.

Moreover, the 'mixed sugars' constituents in the gingerbread are also an important asset because regular sugar intake is essential for proper cognitive function. The presentation in the form of gingerbread is also likely to be more acceptable to people of advancing age who are less keen on taking powders, tablets or capsules because they are often already on medication.

Under European Commission Regulation No. 432/2012 [12], the incorporation of certain minerals and oligo-elements into foodstuffs may give rise to a 'generic' health claim. This is the case for magnesium and vitamin B6 for which the following health claims are made: 'contributes to normal functioning of the nervous system and normal psychological function' and vitamin B5 (pantothenic acid) which it is claimed 'contributes to normal mental performance'. Even so, their inclusion in various food matrices could potentially affect their efficacy. This study was designed to confirm the effect on stress, mood and sleep of four weeks of daily intake of gingerbread enriched in magnesium and vitamins B5 and B6 in the required quantities as determined by EFSA (30\% RDAs).

\section{Material and Methods}

\section{Nature of the study}

Prospective observations study conducted in clinical investigation centre on healthy volunteers.

\section{Primary objective}

The study objectives were to evaluate the effect of daily intake of gingerbread enriched in magnesium and B vitamins on mood, psychological stress and sleep and on satisfaction felt by subjects. 
Citation: $\quad$ Allaert FA, Schueller R, Herpin F, Rigaudier F (2017) Observational Study of the Effect of Consumption of a Magnesium- and B VitaminEnriched Gingerbread on Cognitive Well-being (Mood, Stress, and Sleep). Vitam Miner 6: 162.

Page 2 of 5

\section{Subject selection}

The study was proposed to the CEN Nutriment panel of men and women aged 18-70 years who liked gingerbread, complained of transient stress or low spirits (mood) and possessed a smartphone compatible with the Food Trial ${ }^{\circ}$ secure data collection application. The application uses the data collection engine and cryptographic security of Nurstrial, which has been authorized by the data protection agency, CNIL (DR 915660). To avoid changes in observation conditions, no exclusion criterion was imposed other than not meeting the inclusion criteria and having a known allergy to the product constituents, especially gluten and honey.

\section{Evaluation criteria}

The evaluation criteria were the MSP-9 scale for psychological stress, the Brief Mood Introspection Scale (BMIS), the Spiegel scale for sleep and a six-point Likert scale for quantifying subject satisfaction.

\section{Conduct of the study and data collection}

The first 200 people on the CEN Nutriment panel meeting the selection criteria and wishing to take part in the study were invited to the clinical investigation centre.

After the investigator checked the selection criteria and secured the subject's verbal consent, subjects were given a batch of products and a Food Trial user brochure for installing and using the application on their smartphone. The smartphone application enabled data to be collected and anonymity maintained. Subjects were told it was important to complete the evaluation scales on the smartphone for seven days before beginning to take the product, which would provide an objective vision of their cognitive well-being (humour, stress and sleep) before taking the product. Subjects were given sufficient products for four weeks.

When subjects made their first connection with the application, they described their sociodemographic characteristics. They then filled out the BMIS, MSP-9 and Spiegel scales daily for the first seven days before taking the product (D-7 to D0) and during the final seven days of intake (D21 to D28). The hedonic and satisfactions scales were also completed during the last seven days of intake.

\section{Description of the statistical analysis}

The subjects included in the study were described by means and standard deviations for quantitative variables and by actual numbers and frequency for qualitative variables. Changes in stress, mood and sleep quality were compared between the first and second period using a paired Student's test. Factors influencing change were examined by analysis of variance. This analysis also describes subjects' opinion in terms of satisfaction and taste.

\section{Justification of the number of subjects}

The sample size was calculated on the basis of changes in mood evaluated on the BMIS scale. Based on a mean increase of at least 1.5 points in the BIMS score, with a standard deviation of 2.5 , an alpha risk of 0.05 , power of $90 \%$ and using a paired t-test, the required number is 118 [13]. This number was increased to 200 so as to take into account subjects who were lost sight of. Computations were performed on NQUERY software.

\section{Study product}

The product under study is gingerbread in the form of a $40 \mathrm{mg}$ ration enriched with $110 \mathrm{mg}$ magnesium, $2 \mathrm{mg}$ vitamin $\mathrm{B} 5$ and $0.7 \mathrm{mg}$ vitamin B6, corresponding to $30 \%$ of daily intake for these three constituents. Volunteers were asked to eat 1 ration per day at the time of their choice for the 28 days of the study.

\section{Results}

The study covered 185 people, aged $39.0 \pm 12.0$ years on average ( $\min 20$; $\max 68$ ) $80.5 \%$ of whom were women and whose mean BMI was $24.9 \pm 5.2 \mathrm{~kg} / \mathrm{m}^{2}$.

\section{Changes in stress, mood and sleep}

Before starting intake of enriched gingerbread, volunteers' stress was rated $42.6 \pm 8.5$ on the MSP- 9 scale $(60 \%$ scored 40 or more, indicating substantial stress), mood was rated $38.6 \pm 6.3$ on BMIS and sleep quality $16.2 \pm 3.5$ on the Spiegel scale. Among the subjects, $36.2 \%$ had pathological sleep (less than 15 points), 50.3\% average quality sleep, and $13.5 \%$ very good quality sleep.

By the end of the study, stress intensity had declined significantly from $42.6 \pm 8.5$ to $30.9 \pm 8.4(\mathrm{p}<0.0001)($ Graph 1$)$, that is, a $26 \%$ improvement; the percentage of subjects with substantial stress had fallen significantly from $60.0 \%$ to $14.6 \%(\mathrm{p}<0.001)$ (Graph 2$)$; and an analysis made specifically of the patients suffering most stress showed that its intensity fell from $48.1 \pm 5.8$ to $33.1 \pm 9.3(\mathrm{p}<0.0001)$, corresponding to a $31 \%$ improvement (Graph 3).

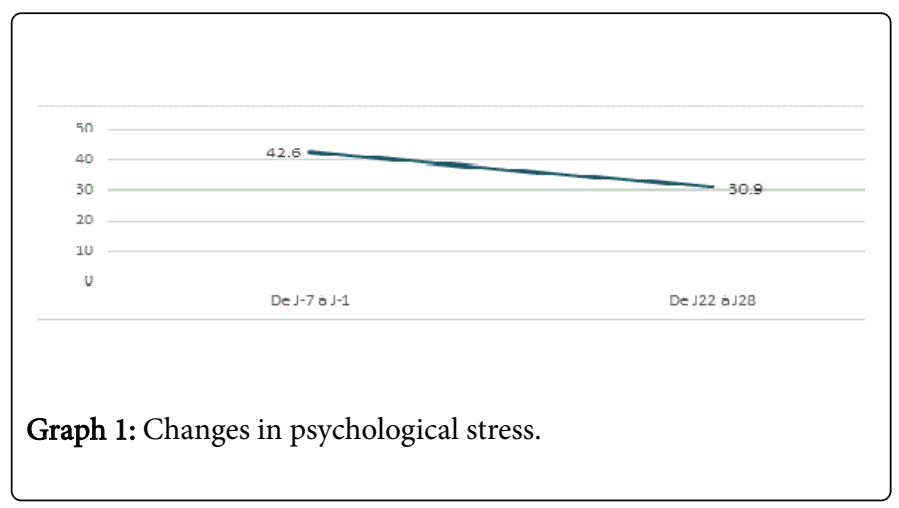


Citation: $\quad$ Allaert FA, Schueller R, Herpin F, Rigaudier F (2017) Observational Study of the Effect of Consumption of a Magnesium- and B Vitamin-

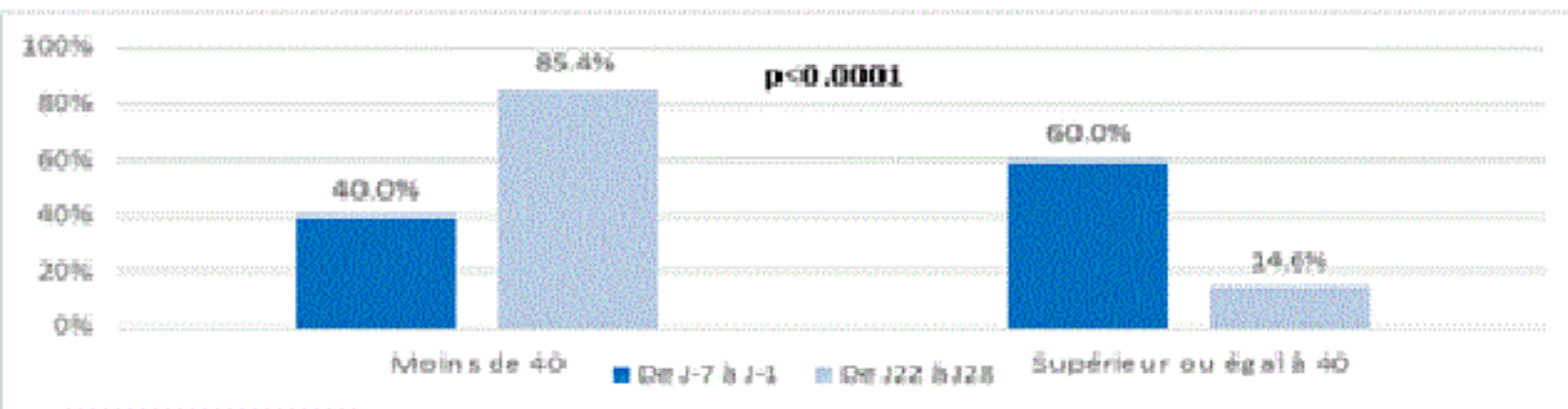

Graph 2: Changes in subjects experiencing intense stress (MSP-9 $\geq 40$ ).

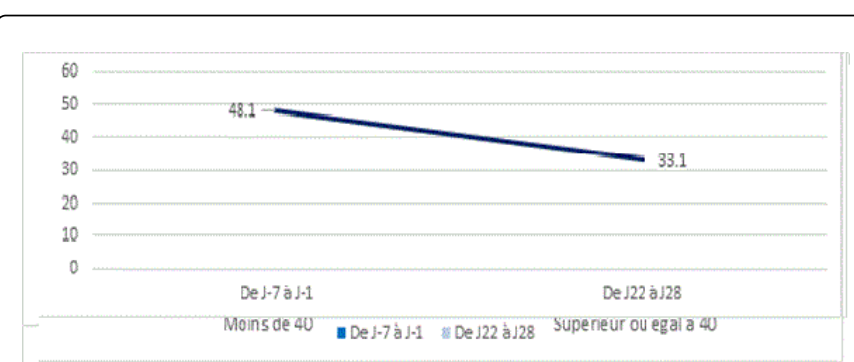

Graph 3: Changes in psychological stress among subjects with MSP-9 $\geq 40$, Mood improved from $38.6 \pm 6.6$ to $47.2 \pm 7.3$ $(\mathrm{p}<0.0001)$, a $25 \%$ progression (Graph 4$)$.

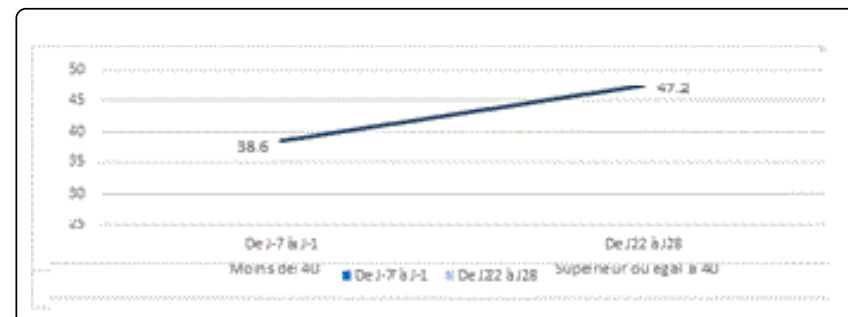

Graph 4: Changes in mood (BMIS), Sleep quality rose from $16.2 \pm$ 3.5 to $19.3 \pm 3.6(\mathrm{p}<0.0001)$, a $23 \%$ improvement (Graph 5$)$.

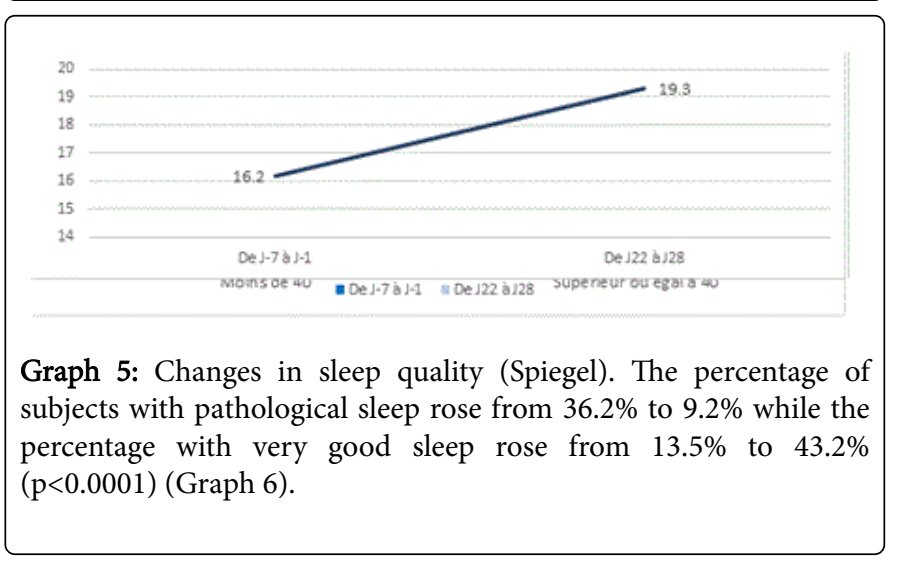

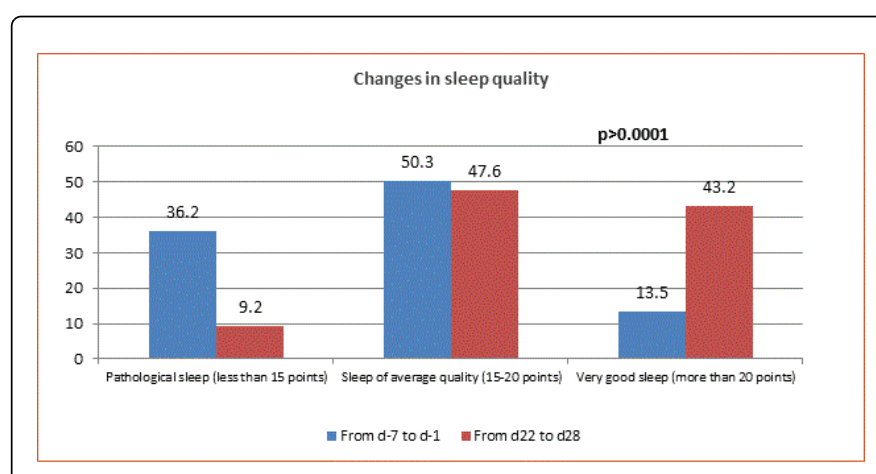

Graph 6: Changes in sleep quality. Subjects said for $35.1 \%$ they were rather satisfied with their cognitive change, $37.3 \%$ satisfied and $13.0 \%$ very satisfied, making a total of $85.4 \%$ positive opinions versus $14.6 \%$ of negative opinions including $10.3 \%$ rather dissatisfied and $4.3 \%$ dissatisfied (Graph 7).

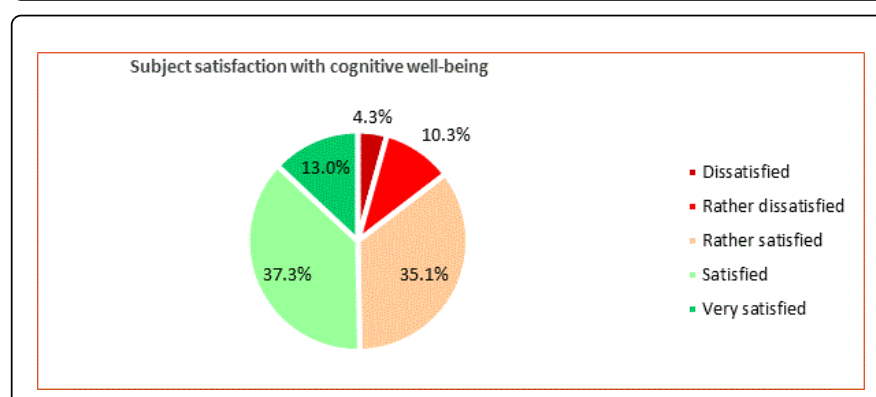

Graph 7: Subject satisfaction with cognitive well-being $\mathrm{p}<0.0001$.

\section{Opinion on the taste of the gingerbread}

Respondents said in $35.1 \%$ of cases the gingerbread tasted rather pleasant, $41.6 \%$ pleasant and $28.6 \%$ very pleasant, giving a total of $93.8 \%$ of positive opinions versus $6.4 \%$ of negative ones including $3.2 \%$ who found the taste rather unpleasant, $0.5 \%$ unpleasant and $2.7 \%$ very unpleasant (Graph 8). 


\section{Appraisal of taste of gingerbread}

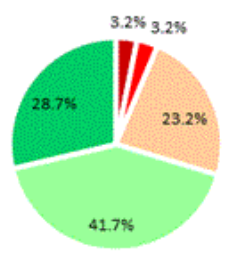

$$
\begin{aligned}
& \text { - Rather unpleasant } \\
& \text { - Rather pleasant } \\
& \text { - Pleasont } \\
& \text { - Very pleasant }
\end{aligned}
$$

Graph 8: Appraisal of taste of gingerbread.

\section{Influence of age and sex}

The study shows no variation in the quality of responses to the supplements as a function of age, sex or BMI.

\section{Discussion}

The results of this real-life study show that the health claims made for magnesium and vitamins B5 and B6 under Commission Regulation No. 432/2012 of 16 May 2012 [12] do indeed have the expected effect on the behaviour of persons who supplement their diet with these components. They also show that the inclusion of these vitamins in a matrix composed essentially of slow and fast sugars and heated during baking does not adversely affect their health benefits.

Traditionally, it is systematically objected to observational studies that they have little probative value as they are not randomized and not conducted blind against a control group. This is true but the purpose here was not to provide proof of the effect of the substances added to the gingerbread, since the claim is already made of magnesium and vitamin B6. It was therefore not a question of repeating the proof but of showing that in actual conditions of everyday use, the inclusion of the products in a matrix of the gingerbread type actually provided subjects with the expected benefits.

The results for stress are of the same magnitude as those observed in the study by Allaert [14] for a dietary supplement enriched in magnesium, probiotics and very many vitamins. That observational study too shows reduced stress of $23 \%$ of 242 subjects, which is similar to the $26 \%$ described in our study. The effect on stress can be attributed to the action of magnesium but also the action of vitamins B5 and B6 through their action on the production of corticosteroids $[15,16]$.

Other work converges with the findings of this study on improved sleep. Abassi [17] shows in a double blind trial versus placebo in elderly people that magnesium supplements significantly increase the duration and quality of sleep and reduce the time needed to fall asleep. The work by Dralle and Bodeker [18] like that by Held et al. [19] shows the existence of a relationship between serum magnesium and slow sleep and that magnesium supplements extend slow sleep cycles and shorten paradoxical sleep cycles. As for the role of lack of magnesium in depressive disorders, it is well established $[20,21]$ but the literature is relatively poor on the associations between vitamins B5 and B6 and mood. In the absence of information, the effect on mood is probably attributable more to the magnesium than to these vitamins.

Lastly, it is important to observe that the addition of these products in a gingerbread structure does not change the taste and that on the contrary the gingerbread arouses considerable gustative interest. This can promote observance among people for magnesium and vitamin B5 and B6 supplements especially those for whom capsules and tablets are too reminiscent of medication or for elderly subjects who already take a lot of medication.

\section{Conclusion}

This observational study conforms that the addition of magnesium and vitamins B5 and B6 in amounts equivalent to $30 \%$ of recommended daily intake produce statistically significant positive effects on stress, sleep and mood. It confirms that the health claim 'contributes to normal functioning of the nervous system and normal psychological function' validated by the EFSA for magnesium and vitamin B6 is still valid when these components are included in a gingerbread type matrix and that subjects are actually able to benefit from them.

\section{References}

1. McEwens BS, Conrad CD, Kuroda Y, Frankfurt M, Magarinos AM, et al. (1997) Prevention of stress-induced morphological and cognitive consequences. Eur Neuropsychopharmacol 3: 323-328.

2. McEwens BS (2001) Plasticity of the hippocampus: adaptation to chronic stress and allostatic load. Ann N Y Acad Sci 933: 265-277.

3. Nelson CA, Carver LJ (1998) The effects of stress and trauma on brain and memory: a view from developmental cognitive neuroscience. Dev Psychopathol 10: 793-809.

4. Bergdahl J, Larsson A, Nilsson LG, Ahlstrom KR, Nyberg L (2005) Treatment of chronic stress in employees: subjective, cognitive and neural correlates. Scand J Psychol 46: 395-402.

5. Stawski RS, Sliwinski MJ, Smyth JM (2006) Stress-related cognitive interference predicts cognitive function in old age. Psychol Aging 21: 535-544.

6. Neupert SD, Almeida DM, Mroczek DK, Spiro A (2006) Daily stressors and memory failures in a naturalistic setting: findings from the VA Normative Aging Study. Psychol Aging 21: 424-429.

7. Lupien SJ, Gaudreau S, Tchiteya BM, Maheu F, Shaima S, et al. (1997) Stress-induced declarative memory impairment in healthy elderly subjects: relationship to cortisol reactivity. J Clin Endocrinol Metab 82: 2070-2075.

8. Magri F, Cravello L, Barili L, Sarra S, Cinchetti W, et al. (2006) Stress and dementia: the role of the hypothalamic-pituitary-adrenal axis. Aging Clin Exp Res 18: 167-170.

9. Hogan MJ (2003) Divided attention in older but not younger adults is impaired by anxiety. Exp Aging Res 29: 111-136.

10. VonDras DD, Powless MR, Olson AK, Wheeler D, Snudden AL (2005) Differential effects of everyday stress on the episodic memory test performances of young, mild-life, and older adults. Aging Ment Health 9: 60-70.

11. Andreoletti C, Veratti BW, Lachman ME (2006) Age differences in the relationship between anxiety and recall. Aging Ment Health 10: 265-271.

12. European Commission. Commission Regulation (2012). establishing a list of permitted health claims made on foods, other than those referring to the reduction of disease risk and to childrens development and health. J Eur Union 50: 1-40.

13. Ho RT, Potash JS, Fang F, Rollins J (2015) Art viewing directives in hospital settings effect on mood. HERD: Health Environments Research \& Design Journal 8: 30-43.

14. Allaert FA, Courau S, Forestier A (2016) Effect of magnesium, probiotic, and vitamin food supplementation in healthy subjects with psychological stress and evaluation of a persistent effect after discontinuing intake. Panminerva Med. 58: 263-270. 
Citation: $\quad$ Allaert FA, Schueller R, Herpin F, Rigaudier F (2017) Observational Study of the Effect of Consumption of a Magnesium- and B VitaminEnriched Gingerbread on Cognitive Well-being (Mood, Stress, and Sleep). Vitam Miner 6: 162.

Page 5 of 5

15. Allgood VE, Powell-Oliver FE, Cidlowski JA (1990) The influence of vitamin $\mathrm{B} 6$ on the structure and function of the glucocorticoid receptor. Ann N Y Acad Sci 585: 452-465.

16. Fidanaza A, Floridi S, Lenti L (1981) Panthenol and glucocorticoids. Boll Soc Ital Biol Sper 57: 1869-1872.

17. Abbasi B, Kimiagar M, Sadeghniiat K, Shirazi MM, Hedayati M, et al (2012) The effect of magnesium supplementation on primary insomnia in elderly: A double-blind placebo-controlled clinical trial. J Res Med Sci 17: 1161-1169.
18. Dralle D, Bödeker RH (1980) Serum magnesium level and sleep behaviour of new-born infants. Eur J Pediatr 134: 239-243.

19. Held K, Antonijevic IA, Kunzel H, Uhr M, Wetter TC, et al. (2002) Oral $\mathrm{Mg} 2+$ supplementation reverses age-related neuroendocrine and sleep EEG changes in humans. Pharmacopsychiatry 35: 135-143.

20. Szopa A, Wlaź P, Nowak G, Radziwoń-Zaleska M, Skalski M, et al. (2013) Magnesium in depression. Pharmacol Rep 65: 547-554.

21. Serefko A, Szopa A, Poleszak E (2016) Magnesium and depression. Magnes Res 29: 112-119. 